\title{
LA DISJONCTION DE LA VOIX NARRATIVE ET LA MANIPULATION DE LA VRAISEMBLANCE DANS LE ROCHER DE TANIOS D'AMIN MAALOUF
}

\author{
Peter Marteinson
}

\section{TORONTO}

This investigation of the narrative voice in Maalouf's Prix-Goncourt winning novel Le Rocher de Tanios observes the manner in which the multiplicity of enunciators, in the form of secondary narrators "cited" intertextually by the primary narrator, engenders a subtle play upon points of view, epochs, and cultural outlooks, an artifice which lends the novel a breadth in its generic status and veridictory grounding. It manages to be both an entirely possible, realistic narrative, and a fantastical legend, in which the "strange and the marvelous", in the words of one of the secondary narrators, form a counterpoint against the rigorous historical research of the primary narrative. The result is a tale in which the appearance of a coherent and inevitable progression of providence melds with a capricious logic of chance events. The work raises the question of fiction and history and answers yes to each one; it is not only a fiction aspiring to verisimilitude, but conversely, it is also an actual history transformed into a novel into the sort of novel that leads the reader to question his sense of truth and falsehood.

On sait qu'il est de nombreux romans qui se présentent comme des documents authentiques, des histoires véridiques, tels Le Paysan parvenu de Marivaux et Don Quichotte de Cervantes. Dans les ouvrages de ce type, surtout à l'aube du genre romanesque, ce travestissement s'explique: certains auteurs semblent avoir eu besoin de dissimuler leur paternité littéraire afin d'éviter la désapprobation de la censure; d'autres auraient tout simplement voulu rendre leurs récits plus vraisemblables.

Dans le cas du Rocher de Tanios, en revanche, on observe un cas presque inverse : il s'agit d'une histoire relevant de la non-fiction que l'auteur, en la transformant en roman, aurait manipulée afin que les traces de son authenticité soient voilées - voire transformées pour ressembler précisément à ces artifices narratifs que l'on emploie pour arrondir la vraisemblance dans le récit de fiction. En d'autres termes, Le Rocher de Tanios est non seulement une histoire vraie déguisée en roman mais, paradoxalement, c'est aussi une de ces fictions «qui se veulent authentiques ». 
Quel est l'aspect «singulier, artificiel et problématique» du récit selon les termes de Genette (1961: 158) - que Maalouf manipule afin d'échafauder les structures «authentiques» de ce roman? Il y en a plusieurs, mais c'est essentiellement grâce à la disjonction de la voix narrative que ce roman réussit, en dépit d'un certain désordre structural, à intégrer deux genres de discours autrement inconciliables : c'est à la fois un texte historique, dont la vraisemblance est convaincante, et une épopée littéraire dans laquelle la légende - le merveilleux, l'étrange - a survécu aux faits, «comme l'âme après le corps, ou comme le parfum dans le sillage d'une femme $»^{1}$.

Dans ce travail on se limitera donc à examiner les différents recours de l'auteur aux multiples instances de la narration, et on tentera d'identifier un certain nombre des significations issues de cette fantasmagorie de la corroboration historique. Car, en multipliant les voix narratives, Maalouf a su manipuler - à tout moment de l'intrigue - la focalisation du discours narratif ainsi que le degré de sa vraisemblance. De la sorte il a pu mettre en marche un jeu subtil de points de vue et d'époques qui semble intégrer les péripéties les plus menues à une logique de la Providence.

Le Rocher de Tanios est avant tout un récit « vraisemblable». Maalouf n'abandonne jamais le souci de préserver la crédibilité de l'histoire: le narrateur, vers le début du roman, ne manque pas de justifier l'ampleur du savoir qu'il a su accumuler au sujet de Tanios, dont l'histoire s'est déroulée au $\mathrm{XIX}^{\mathrm{e}}$ siècle. Non seulement a-t-il recours à des «documents » écrits, mais aussi aux souvenirs (exagérés) d'un des vieillards du village :

Je dois à Gébrayel d'avoir acquis très tôt l'intime conviction que Tanios avait bien été, par-delà le mythe, un être de chair. Les preuves sont venues plus tard, des années plus tard. Lorsque, la chance aidant, je pus enfin mettre la main sur d'authentiques documents.

Il en est trois que je citerai souvent. Deux qui émanent de personnages ayant connu Tanios de près. Et un troisième plus récent. Son auteur est un religieux décédé au lendemain de la Première Guerre mondiale, le moine Elias de Kfaryabda - c'est le nom de mon village, je ne pense pas l'avoir mentionné encore. [p. 13]

Le narrateur qualifie ce livre du moine Elias de la manière suivante: « [... ] étrange, inégal, déroutant. Certaines pages, le ton est personnel, la plume s'échauffe et se libère [... ] et puis soudain, comme s'il craignait d'avoir péché par orgueil, le moine se rétracte, s'efface [ ... ]» (p. 14). Ce

\footnotetext{
${ }^{1}$ Le Rocher de Tanios, p. 265.
} 
personnage du moine est développé non seulement par la description, mais son caractère est activement démontré par l'entremise de l'action. Dans le passage suivant, sa dévotion, ainsi que sa fascination «anthropologique » se manifestent :

Puisse le Très-Haut m'accorder Son pardon pour les heures et les journées que je vais devoir dérober au temps béni de la prière et des Saintes Lectures afin d'écrire cette histoire imparfaite des gens de ma contrée, mon excuse étant qu'aucune des minutes que nous vivons n'aurait existé sans les millénaires qui l'ont précédée depuis la Création, et qu'aucun de nos battements de coeur n'aurait été possible s'il n'y avait eu les générations successives des aïeux, avec leurs rencontres, leurs promesses, leurs unions consacrées, ou encore leurs tentations.

Préambule de la Chronique montagnarde,

Euvre du moine Elias de Kfaryabda [p. 17]

On abordera plus tard le développement au niveau actantiel du Pasteur Stolton et de Nader le muletier, lors de l'examen de leurs «documents » qu'ils nous fournissent.

On pourrait conclure a priori que les « sources» sont exploitées, en gros, lorsqu'il y a une sorte d'ellipse qu'il faudrait élucider; mais, comme on le verra, l'importance de ces «documents authentiques» est souvent d'un tout autre ordre. Vers le début du roman, le narrateur cite l'une de ses sources comme pour expliquer sa curiosité : c'est à la Chronique montagnarde qu'il attribue son inspiration. Cependant, on notera qu'il s'agit en même temps d'une invite au lecteur d'épouser la perspective du narrateur:

« Du quatre novembre 1840 date l'énigmatique disparition de Tanios-kichk... Pourtant, il avait tout, tout ce qu'un homme peut attendre de la vie. Son passé s'était dénoué, la route de l'avenir s'était aplanie. Il n'a pu quitter le village de son plein gré. Nul ne peut douter qu'une malédiction s'attache au rocher qui porte son nom ».

A l'instant, les mille pages cessèrent de me paraitre opaques. Je me mis à regarder ce manuscrit d'une toute autre manière. Comme un guide, un compagnon. Ou peut-être comme une monture.

Mon voyage pouvait commencer. [Op. cit., p. 15]

Cet enclenchement du récit correspond plus qu'à un simple commencement par la fin ou à un regard neuf sur le tome épais du moine en question. En effet, Maalouf sépare «le temps de l'histoire» du «temps de la narration »-qui non seulement évoque une époque bien après celle de Tanios, mais qui semble avoir sa propre durée, en tant que projet de «voyage ». On verra que cette dualité des temps demeure, d'un bout à 
l'autre du roman, une des raisons pour la multiplicité des interprétations, dont l'une relève de la contemporanéité (temps de la narration), et l'autre du moment quand se déroule le récit (temps de l'histoire).

La citation des «documentations » sert donc, dans ce cas, non seulement à situer le narrateur vis-à-vis de l'époque du récit tout en établissant la nature de la relation narrateur-lecteur, mais aussi à investir l'acteur du moine d'un rôle actantiel ${ }^{2}$. En effet, Elias est un actant en tant que destinateur (il provoque chez le narrateur le désir de partir en ce voyage historique) et est également adjuvant, au niveau de la modalité du savoir (parce qu'il aide ce chercheur à clarifier certains faits).

Le narrateur lui aussi a un rôle actantiel (non-figurativisé) dans le schéma des personnages «actuels» (dont le lecteur et Gébrayel) : d'abord, c'est un archéologue qui a dû chercher rigoureusement, méthodiquement et d'une façon scientifique, pour découvrir l'essentiel de l'histoire ; dans ce rôle il est perspicace, sceptique et très adroit (compétences relatives à la modalité du savoir). Ensuite, comme il a déjà été suggéré, le narrateur est une sorte de bavard qui tend à s'écarter de l'histoire pour relater d'autres épisodes apparemment moins pertinents que le récit principal. A titre d'exemple, dans l'explication initiale de références historiques qu'il évoque, celui-ci commence à décrire le village de Kfaryabda; mais très vite il finit par décrire la beauté d'une de ses femmes (Lamia). Alors, à partir de cette digression, il parvient à nous brosser un portrait du cheikh Francis, le seigneur du village. Et comme un des "petits défauts » du cheikh est, pour ainsi dire, ce qui amorce le récit, il ne suffit pas de simplement nommer ce défaut; il faut, comme pour augurer une malédiction, qu'on l'explique en détail. Toutefois, ce n'est pas seulement le narrateur, mais aussi le vieillard sus mentionné, qui l'évoque :

[... ] Les gens de Kfaryabda considéraient «leur » cheikh comme un moindre mal. Il serait même apparu comme une véritable bénédiction s'il n'avait eu un travers, un insupportable travers qui, aux yeux de certains villageois, réduisait à néant ses plus nobles qualités.

- Les femmes! me dit le vieux Gébrayel, et dans son visage de buse s'allumèrent des yeux carnassiers. Les femmes! Le cheikh les convoitait toutes, et il en séduisait une chaque soir !

S'agissant du dernier bout de phrase, c'est une affabulation. Mais pour le reste $[\ldots]$ [... 29]

\footnotetext{
${ }^{2}$ Pour une explication du schéma actantiel, voir Greimas, Du sens II : Essais sémiotiques, pp. 49-65.
} 
Une nouvelle fois, l'utilisation d'une autre voix narrative n'a d'autre utilité que celle d'informer le lecteur. Aux yeux de ce dernier, Gébrayel, lui aussi, commence à avoir une personnalité, ainsi qu'une dimension actantielle, et la diégèse des «modernes» se développe de nouveau. La structure romanesque se nuance - ou plutôt se redéfinit - au fur et à mesure que se déroule cette première partie de l'ouvrage. Ainsi les ruptures provoquées par les différentes voix narratives, au lieu de compromettre le déroulement du récit, se manifestent plutôt en tant que véhicules sémantiques parallèles. Cette situation rappelle une observation de Georges Poulet à l'égard de la sémantique de la forme : "C'est le propre de l'œuvre à la fois d'inventer ses structures et de les dépasser $\gg .^{3}$

Peu après, une rupture narrative enclenchée par Gébrayel constitue un apport qui fait évoluer la structure du récit ainsi que sa densité sémantique, plus qu'elle ne détruit l'illusion de la cohérence narrative. Il s'agit du commentaire au sujet des femmes du village, et de leur relation avec le cheikh :

La plupart, toutefois, et quoi qu'en dise le vieux Gébrayel, ne voulaient pas de ces amours compromettantes et sans lendemain. Elles ne se prêtaient à aucun autre jeu galant que la dérobade, et il semble bien que le maître savait s'y résigner lorsque son « adversaire» se montrait futée. [p. 25]

Le narrateur semble vouloir prévenir le lecteur contre la (fausse) possibilité que ce dernier aurait pu recueillir, lors de quelque autre occasion extradiégétique ${ }^{4}$, une information inexacte de la part du vieil homme. Ce qui signifie que le narrateur, dans l'imaginaire qu'il partage avec le lecteur, amplifie les dimensions spatiales et temporelles de la «lecture» à un tel degré que celles-ci, plutôt que de s'articuler comme un simple fil linéaire qui réunit les épisodes de l'intrigue, se manifestent en tant qu'ensemble d'événements parmi d'autres. Si le moine Elias est le destinateur de «la quête des recherches historiques » du narrateur (un " actant-chercheur »), on pourrait dire qu'au moyen d'une représentation implicite d'une autre structure actantielle le lecteur, lui aussi, devient un destinateur. Au fur et à mesure que le narrateur dévoile qu'il se soucie de sa compréhension, celui-ci s'avère être à l'origine du désir du «chercheur» de devenir «conteur». Ainsi, le temps «des recherches» pen-

\footnotetext{
${ }^{3}$ Poulet, G., La Distance intérieure. Voir aussi Umberto Eco, La Struttura assente, p. 323.

${ }^{4}$ En dehors de la diégèse principale (l'univers de narration principale); cf. Genette Figures III.
} 
dant lequel le narrateur a dû étudier les documents historiques se distingue encore plus du temps de la narration qui se réalise non seulement logiquement, mais aussi textuellement, après ce temps des recherches, qui lui aussi suit le temps du récit.

C'est la séduction par le cheikh de la femme de son intendant qui est l'événement fatal de l'intrigue, celui qui déclenche toute une série de conséquences de plus en plus graves. Il s'agit de Lamia, la plus belle femme du village, et à cause de cette liaison interdite, elle va enfanter un fils dont tout le monde soupçonne l'illégitimité : Tanios. Ce qui est notable du point de vue critique, c'est que le narrateur «n'a pas d'autre choix » que de recourir à la version populaire de cet événement, telle que racontée par Gébrayel. Effectivement, tout au long du passage en question, on ne sait pas trop si c'est ce dernier qui parle, ou s'il s'agit de l'interprétation du narrateur. Or, Maalouf ne manque pas de profiter de l'occasion que présente cette intervention de Gébrayel pour parvenir à ses fins :

[... ] Les femmes de ce temps-là dévoilaient leur poitrine sans le moindre soupçon d'indécence, et Lamia laissait paraître une face entière de chaque sein. Sur ces collines-là j'aurais voulu poser ma tête chaque nuit...

Je m'éclaircis la gorge.

- Comment peux-tu savoir tant de choses, tu ne l'as jamais vue !

- Si tu ne veux pas me croire, pourquoi m'interroger?

Mon intrusion dans son rêve l'avait irrité. Mais il ne m'en tint pas rigueur. Il se leva, prépara pour lui et pour moi deux grands verres de sirop de mûre.

- Bois lentement, me dit-il, l'histoire est encore longue. [p. 38]

On retrouve de nouveau la réalisation d'un événement extradiégétique qui est loin d'être gratuit. Ce passage contribue non seulement à développer les caractères du narrateur et de Gébrayel, ainsi que leur schéma actantiel mais, de plus, il réussit à accentuer bien plus le «point culminant» de la séduction, par une sorte de renversement qui fait, en sorte, que la rupture, en tant qu' «accident» devient une espèce de preuve d'authenticité. Le récit en est alors plus convaincant à un niveau purement psychologique et imaginaire : c'est une pause avant le paroxysme, un silence qui précède l'orage.

C'est grâce à un phénomène similaire que la grossesse qui résulte de cette liaison semble plus fatale, plus dangereuse encore. Si un peu plus tard dans le récit le fil de l'histoire est de nouveau rompu ostensiblement à cause de la découverte d'un document authentique, c'est aussi pour «choquer par pudeur» le narrataire, que le narrateur n'évoque pas en premier la naissance de Tanios. Cette ellipse vis-à-vis de son énoncé 
accentue l'importance de l'événement, qui semble s'imposer au narrateur avant qu'il n'ait le temps d'y arriver de son propre gré. Ceci est d'autant plus vrai que le passage suivant est mis en exergue au début d'un nouveau chapitre :

En l'année 1821, vers la fin du mois de juin, Lamia, épouse de Gérios, l'intendant du château, donna naissance à un garçon, qu'on prénomma d'abord Abbas, puis Tanios. Avant même d'ouvrir ses yeux innocents, il avait attiré sur le village un torrent de malveillance imméritée.

C'est lui qui, plus tard, fut surnommé kichk, et connut le destin que l'on sait. Sa vie entière ne fut qu'une succession de passages.

\section{Chronique montagnarde,}

CEuvre du moine Elias de Kfaryabda [p. 45]

Effectivement, cette épigraphe pose une question évidente au sujet des répercussions possibles qui pourraient avoir lieu à la suite de la naissance de ce garçon; cet être tellement innocent et aucunement responsable de ce qui s'est produit. Le lecteur remarque, une nouvelle fois, ce passage de roman ne semble être qu'une simple analepse ${ }^{5}$ par rapport à l'Histoire, qui mènera au « destin que l'on sait».

Peu après, au moment quand on choisit le nom de l'enfant, la rupture de l'énoncé narratif fait ressortir le thème de l'actant du destin, qui est peu nocif et qui a le pouvoir, à cause de son savoir profond de la nature humaine, de profiter d'un événement de prime abord insignifiant, et d'en faire une péripétie bouleversante. Ainsi, Maalouf fait jouer la dialectique qui oppose l'homme et la Providence, et le lecteur ne peut pas s'empêcher de ressentir cette idée. Ici, le cheikh suggère au «père » de donner à l'enfant l'un des prénoms les plus prestigieux de sa propre famille. L'intendant n'a pas d'autre choix si ce n'est que d'approuver le prénom, «Abbas ». Mais tout de suite après, tous se rendent compte que le cheikh $\mathrm{y}$ avait pensé par orgueil car il se croyait le père biologique probable de l'enfant :

Promenant son regard réjoui sur l'assistance pour recueillir les approbations d'usage, Gérios remarqua que le curé avait les sourcils froncés, et que la cheikha s'était mise soudain à serrer son enfant contre elle avec une rage incompréhensible. Elle était blême comme une branche de curcuma, [ ... ]

Les yeux de Gérios s'attardèrent un moment sur elle. Et soudain, il comprit. $[\ldots]$

La scène n'avait duré qu'une pincée de secondes, mais pour l'enfant, pour

\footnotetext{
${ }^{5} \mathrm{La}$ prolepse représente un moment dans le futur, tandis que l'analepse reprend et réexamine un moment du passé (Genette).
} 
ses proches, pour le village entier, tout avait soudain basculé. «Ce jour-là, écrit l'auteur de la Chronique montagnarde, leur destin à tous fut consigné et scellé ; comme un parchemin il n'aurait plus qu'à se dérouler ». [p. 49]

Peu importe si la femme du curé, la soeur de Lamia, a pu s'arranger pour changer le choix du prénom au profit de «Tanios»; le garçon, bien qu'il passera ses premières années sans entendre parler du bruit qui circulait à l'égard de sa paternité, ne tarde pas trop à comprendre qu'il est «bâtard », et que tout le monde sauf lui en était conscient depuis le jour de sa naissance.

C'est « le fou du village », Challita, qui le lui révèle, devant un groupe de jeunes garçons. Ici, encore une fois, l'événement est accompagné d'une « citation » qui porte une signification d'ordre thématique :

«A chaque époque, commente le moine Elias, il s'est trouvé parmi les gens de Kfaryabda un personnage fou, et lorsqu'il disparaissait, un autre était prêt à prendre sa place comme une braise sous la cendre pour que ce feu ne s'éteigne jamais. Sans doute la Providence a-t-elle besoin de ces pantins qu'elle agite de ces doigts pour déchirer les voiles que la sagesse des hommes a tissés ». [p. 77]

Encore une fois, on évoque une espèce de lutte entre l'homme et le destin, entre l'homme et son Dieu.

Ce moment du récit marque un point de transition important: Tanios, ayant perdu sa naïveté, s'écarte de plus en plus des autres villageois. L'exergue qui ouvre le "Quatrième passage », et qui s'intitule "L'école du pasteur anglais », remplit la fonction évidente de charnière dans la logique de l'intrigue. Toutefois, il s'agit de nouveau d'une achronie qui anticipe et présage l'avenir, car la citation est un extrait d'une lettre que le narrateur a reçue du directeur actuel de cette école anglaise :

Je suis heureux de vous confirmer, en réponse à votre lettre, qu'il y avait bien, parmi les tout premiers élèves de l'école de Sahlaïn, un dénommé Tanios Gérios, de Kfaryabda. Le fondateur de notre établissement, le révérend Jeremy Stolton, était venu s'installer dans la Montagne avec son épouse au début des années 1830. Il existe, dans notre bibliothèque, un petit coffret où sont conservées ses archives, notamment, pour chaque année, des éphémérides parsemées d'annotations diverses, ainsi que des lettres. Si vous souhaitez les consulter, vous êtes le bienvenu, mais vous comprendrez qu'il ne puisse être question pour nous de les laisser sortir...

Extrait d'une lettre du révérend Ishaac, directeur actuel de l'École anglaise de Sahlaïn. [p. 101]

Le narrateur va pouvoir profiter, désormais, d'une nouvelle source primaire, artifice que ce passage subtil a comme fonction d'introduire et de 
justifier. C'est pendant sa vie adulte que Tanios va aussi connaître le personnage de Nader, le muletier, qui lui aussi vit presque en marge de la société villageoise. Le narrateur a expliqué, un peu avant le dernier passage cité, que Nader était un marchand très intelligent, mais assez étrange, qui aimait lire et écrire, mais qui n'appréciait guère la compagnie des gens «normaux ». Comme d'habitude, Maalouf ne tolère aucune rupture du fil de l'énoncé narratif sans qu'il y ait au moins deux raisons de le faire. Le passage suivant, à part sa fonction d'introduire Nader, se manifeste sous forme d'une référence proleptique ${ }^{6}$ renouvelée à la fin de l'histoire connue de Tanios :

À propos de cet homme, je n'ai pas encore dit le plus important: il est l'auteur du seul ouvrage qui renferme une explication plausible de la disparition de Tanios-kichk. [ ... ]

Sans doute ces écrits n'étaient-ils pas destinés tels quels à la publication. C'est en tout cas bien après la mort de Nader qu'un universitaire les a retrouvés et édités sous un titre que j'ai traduit par « la Sagesse du muletier»; j'aurai souvent recours à ce précieux témoignage. [p. 89]

La scène est donc préparée pour les derniers «passages » du roman, qui sont de nature assez différente de celles qui les précèdent. Car Tanios, à l'aide de l'éducation qu'il a reçue à l'école du révérend Stolton, va jouer un rôle d'une importance surprenante dans la politique régionale. Il s'agit de l'époque pendant laquelle l'Égypte s'engage avec la France dans une guerre contre l'empire ottoman et ses alliés, la Russie et l'Angleterre.

Tout comme l'actant du narrateur a une fonction secondaire de «mettre en question » les énoncés du vieux Gébrayel, le révérend Stolton, un Anglais, médiatise un point de vue à la fois plus «moderne» et plus occidental que l'ensemble des autres personnages (tous originaires de la Montagne, la région du Liban actuel). Non seulement le pasteur est-il une source d'informations au sujet de Tanios, il est aussi l'instrument de l'auteur pour donner au lecteur une perspective sceptique pour interprétation de l'intrigue. Donc, l'acteur de Stolton, dans lequel s'investit un rôle actantiel important dans l'imaginaire des contemporains de Tanios (en tant que destinateur dans le développement intellectuel de celuici) a également une fonction importante, à travers ses écrits, vis-à-vis de l'imaginaire «moderne». Cet Européen est un acteur sur lequel le lecteur peut «projeter »- au sens freudien du terme - ses doutes. C'est pour cette raison qu'il est un pilier important pour assurer l'intégrité et la cré-

\footnotetext{
${ }^{6}$ Ici proleptique signifie un regard momentané sur un événement futur.
} 
dibilité de l'histoire. L'épisode du blanchissement soudain de la chevelure de Tanios, qui s'effectue à l'âge de dix-sept ans, contribue à la dimension fantastique du Rocher de Tanios. Mais cet épisode aurait risqué de compromettre le récit sans la citation suivante du journal intime du pasteur:

J'ai cru comprendre toutefois qu'il existe dans ce coin de la Montagne une légende concernant des personnages à la chevelure prématurément blanchie, qui, depuis l'aube des temps, apparaitraient épisodiquement en certaines périodes troubles, pour disparaître aussitôt. On les appelle des «vieilles-têtes », ou encore des «sages-fous». Selon certains, il s'agirait même d'un personnage unique qui se réincarnerait indéfiniment. Il est vrai qu'en pays druze la métempsycose est une croyance solidement établie.

\section{Éphémérides du révérend Jeremy Stolton,} Année 1836 [p. 127]

On remarque justement combien le lexique scientifique de Stolton semble rendre l'événement plus crédible. De plus, la mention du «pays druze », qui souligne le caractère étranger de cette région aux yeux du pasteur, soulage les doutes du lecteur - pour qui la distance culturelle avec ce qui est décrit peut augmenter l'écart temporel et factuel de cette partie de l'intrigue.

La fonction d'autorité scientifique de cet actant n'est pas plus importante que son rôle dans la formation morale de Tanios. Car ce dernier trait de caractère du révérend Stolton, renforcé par le fait qu'il inspire confiance au lecteur, sert à médiatiser une critique de certains personnages "d'extrême gauche" que le Maalouf aurait difficilement trouvé le moyen d'exprimer par l'entremise du narrateur. Lors de la scène d'une réunion de soldats égyptiens à laquelle assiste le jeune Tanios, dans la maison d'un personnage « révolutionnaire», un long énoncé du narrateur est rompu par une espèce d'interjection de la part de l'historien objectif qu'est Stolton :

«Rien moins qu'une réunion de famille», ironise le pasteur Stolton dans un compte rendu détaillé de cette rencontre, compte rendu inspiré de ce que son pupille - pour une raison qu'on ne va pas tarder de comprendre - allait lui rapporter à l'instant même de son retour à Sahlaïn. [p. 143]

Le personnage du muletier, Nader, est également investi d'une polyisotopie actantielle. Si son journal intime permet parfois au narrateur de rapporter certains événements généralement oubliés, son importance principale est celle de médiatiser les réflexions les plus globales, les plus poétiques de l'auteur. La citation suivante est d'une phrase de Nader que 
le narrateur met en exergue lors d'un nouveau chapitre. Cet exemple fait ressortir davantage le thème «historico-philosophique» de la raison de l'homme, ou plutôt son peu de raison :

La parole du sage s'écoule dans la clarté. Mais de tout temps les hommes ont préféré boire l'eau qui jaillit des grottes les plus obscures. [p. 71]

Le meurtre du patriarche est l'événement le plus important du roman. Celui-ci est l'un des hommes les plus vénérés de la Montagne, qui se trouve victime de Gérios, le père de Tanios (ou plutôt le mari de sa mère...). Ce meurtre, pourtant, ne provoque aucune surprise chez le lecteur, qui voit l'épisode à la fois comme un « fait divers » historique, déjà très connu, et un acte abominable qui aurait dû choquer toute la Montagne. La raison de cette double perspective se trouve dans la relation prolepse/analepse entre l'épigraphe suivante et l'énoncé du narrateur après dans le texte du roman:

Il était écrit que les malheurs qui ont frappé notre village devaient culminer en un acte abominable, portant malédiction: le meurtre du patriarche triplement vénéré, par des mains qui ne semblaient pourtant nullement faites pour le crime.

\section{Chronique montagnarde,}

Euvre du moine Elias de Kfaryabda [p.149]

L'évocation par le moine d'un langage de la Providence semble paradoxalement dramatiser l'événement. De plus, l'écart temporel que crée la dualité des énoncés (temps de la narration/temps du récit) rend le crime de Gérios d'autant plus pesant qu'il a pu survivre dans la mémoire populaire pendant une période si longue.

La fonction justificatrice des trois «documents» semble donc, à ce stade assez avancé du récit, être devenu superflue. Maalouf est confiant que le lecteur sera satisfait en ce qui concerne la vraisemblance, car celle-ci n'est plus guère accentuée. La focalisation d'une certaine partie du récit porte sur la pensée du meurtrier, Gérios - en dépit du fait que celui-ci va mourir sans laisser aucun manuscrit expliquant la manière dont l'embuscade s'est déroulé. L'inclusion de petits détails contribue à dramatiser le récit mais aurait pu toutefois rompre l'illusion romanesque : "Gérios porta l'arme en la serrant tout contre lui. Elle avait une odeur de résine chaude» (p. 165). Ce qui est intéressant, c'est que la Chronique montagnarde est citée une nouvelle fois quelques pages plus loin, comme pour rassurer le lecteur que les faits que l'on rapporte ne sont pas seulement des reconstructions hypothétiques. Tanios, quelques minutes après le meurtre, vient de retrouver son père et décide de s'enfuir avec lui : 
Son forfait accompli, l'intendant Gérios s'empressa de dévaler la colline en compagnie de son fils. Ils se dérobèrent aux regards, et le cheikh dut renoncer à les faire poursuivre.

Arrivés au fond de la vallée, ils marchèrent jusqu'à la tombée de nuit et encore toute la nuit, au voisinage du torrent, vers la mer. [p. 174]

Les deux hommes accostent à Famagouste, en Chypre, où ils passent en exil les douze prochains mois. C'est là où Tanios va rencontrer Thamar, une prostituée géorgienne qui devient son amante. Aussi il fait dans cette ville maritime la connaissance de M. Hovsépian, le drogman du consul de l'Angleterre, qui promet au révérend Stolton de chercher son ancien élève, et d'essayer d'assurer sa sécurité.

Si la Chronique montagnarde constitue en gros la voix des faits historiques (de la documentation), la voix narrative de Nader est celle qui contribue le plus, comme on l'a mentionné, à l'élément merveilleux du roman, élément qui se manifeste de plus en plus souvent dans cette dernière partie. Dans une sorte de «nostalgie en prolepse», on cite Nader pour annoncer la triste fin de cet amour entre Thamar et Tanios: "Quand le bateau t'attendait au port, tu l'as cherchée pour lui dire adieu. Mais de cet adieu-là, ton amante ne voulait pas ». [p. 198]

Quelque douze mois après la fuite de Gérios et de son fils, un agent de l'émir de la Montagne, ayant entendu dire qu'ils se cachaient à Famagouste, réussit à tromper Gérios. Il lui a fait croire que l'émir ait été défait, que la guerre était finie et que l'on avait prononcé une amnistie générale. Gérios est alors exécuté par pendaison dès son retour au Liban.

Une nouvelle fois, le côté choquant de toute cette histoire de guerre se fait ressentir non pas par le recours à des descriptions grotesques, mais par un moyen presque inverse de ruptures du fil de l'histoire. Ce qui se manifeste sous forme d'une distanciation, d'une pudeur et d'un temps de réflexion qui accentue l'impression chaotique de l'époque :

Ce n'est pas ainsi que j'aurais dû présenter la grande révolution sociale qui s'est produite dans mon village en ce temps-là. Pourtant, c'est ainsi que mes sources la révèlent, et c'est ainsi qu'elle est restée dans la mémoire des vieillards.

Peut-être aurais-je dû maquiller un peu les événements, comme d'autres ont fait avant moi. J'aurais sans doute gagné en respectabilité. Mais la suite de mon histoire en serait devenue incompréhensible. [p. 221]

On remarque combien l'évocation des sources, y compris celle de «la mémoire des vieillards », semble mettre en place une perspective qui enclenche à priori une appréciation plus profonde de l'épisode en question.

Au moment de la véritable défaite de l'émir, Hovsépian suggère au 
Consul anglais d'engager Tanios, parce qu'il est un fils du pays, à traduire et à lire publiquement (devant l'émir) le constat du bannissement de ce monarque. A cause d'une confusion d'interprétations, une conversation suit cette proclamation, et le représentant du Sultan turc insiste pour que l'émir soit exilé à Istanbul. L'émir, ayant compris qu'une telle fin signifierait sa mort, supplie à Tanios, à voix basse, de «dire quelque chose », et cela en dépit du fait que c'est l'émir qui avait condamné Gérios à la pendaison.

«Et l'enfant de Kfaryabda, rapporte la Chronique, écoutant la parole du vieillard humilié, écarta son désir de vengeance comme il l'avait déjà mille fois assouvi, et dit à voix haute : "Son Altesse pourrait aller à Malte !" ». [p. 249]

Ce que le Consul anglais accepte immédiatement. De cette manière, Tanios est reconnu comme un héros. Le narrateur, qui fait appel à la documentation pour décrire la suite de cet événement, contribue à rendre l'étrange récit plus vraisemblable :

Par un étrange malentendu - ou disons par une forme d'exagération fanfaronne à laquelle les Orientaux ne répugnent pas -, on attribue à Tanios le rôle le plus éminent qui soit, celui d'avoir chassé l'émir du pays tout en lui gardant généreusement la vie sauve, comme si les Puissances européennes et l'Empire ottoman, avec leurs armées, leurs flottes, leurs diplomates et leurs agents, n'avaient été que de modestes comparses dans un bras de fer théâtral entre l'enfant prodige de Kfaryabda et le despote qui avait condamné son père. [p. 253]

Le narrateur s'approche donc d'un point de vue occidental (celui du lecteur français ?) au moyen de l'artifice des éphémérides du révérend Stolton, qui décrivent la surprise de ce dernier à propos de ces "Orientaux » et qui arrive ainsi à désamorcer, pour ainsi dire, les doutes du lecteur.

Eu égard à sa structure ambiguë, il est peu surprenant que le roman se termine sur une note d'incertitude. Si Maalouf a voulu juxtaposer le mystérieux avec l'illusion d'une certaine historicité, néanmoins, il n'a pas pu résister à une dernière apologie de la qualité inouïe de l'événement final :

Sous cette lueur incertaine commença le dernier jour dans l'existence de Tanios-kichk - son existence connue, tout au moins. Je me trouve cependant contraint d'interrompre sa course et de revenir en arrière pour évoquer à nouveau sa nuit dernière. J'ai tenté de la restituer du mieux que je pouvais. Il existe cependant une autre version de la même nuit. Que rien dans les sources écrites ne vient corroborer et qui - chose plus grave selon mes critères - n'a pas non plus le mérite de la vraisemblance.

Si j'en parle quand même, c'est que le vieux Gébrayel m'en voudrait si je 
l'omettais ; je me rappelle encore à quel point mes doutes l'avaient irrité. « Rien qu'une légende, dis-tu? Tu ne veux que les faits? Les faits sont périssables, crois, moi, seule la légende reste, comme l'âme après le corps, ou comme le parfum dans le sillage d'une femme.» Je dus lui promettre de mentionner sa variante. [p. 265]

Et le roman s'avère avoir deux fins. Selon l'une, Tanios s'endormit pour aller le lendemain parler avec Nader avant de partir pour une destination qui n'est pas précisée. Selon l'autre, l'ancienne petite amie de Tanios, Asma, vient passer cette nuit finale dans ses bras, puis le lendemain, après sa conversation avec Nader, il va s'asseoir sur le rocher en forme de trône qui portera son nom, et il disparaît.

Cependant, un passage étrange et ambigu de « la Sagesse du muletier» donne, pour les lecteurs sceptiques, une version plus vraisemblable, bien qu'elle consiste en phrases énigmatiques et symboliques :

Pour tous les autres, tu es l'absent, mais je suis l'ami qui sait. À leur insu tu as couru sur le chemin du père meurtrier, vers la côte.

Elle t'attend, la fille au trésor, dans son île; et ses cheveux ont toujours la couleur du soleil de l'occident. [p. 276]

Le Rocher de Tanios (prix Goncourt 1993) est un roman, il faut le conclure, qui dépasse ses structures. C'est à la fois un récit de ce «style oral» qui caractérise la littérature arabe actuelle, un ouvrage basé sur le modèle de notre roman moderne, et un «roman fantastique » qui provoque l'imagination du lecteur. Sa structure rappelle un commentaire de Rousset (1962 : 49-50) au sujet d'un procédé narratif de Marivaux, qui

interrompt sans cesse la narration à la 3 e personne pour se projeter au premier plan, $[\ldots]$ jugeant et interprétant ses personnages, leurs actes et leur langage. C'est un livre dans le livre et sur le livre [...] qui est aussi une esthétique du bâton rompu, de la variété, du « hasard».

La disjonction de la voix narrative, la séparation du temps du récit et du « temps de l'énoncé du narrateur » et la manipulation d'une optique tantôt vraisemblable, tantôt merveilleuse - ces éléments qui font le style tout à fait unique de ce roman - peuvent résulter, comme on l'a vu, en grande mesure de l'emploi très adroit des fausses « sources » qui forment le sujet de cette investigation. On peut dire que cette "épaisseur de signes », pour emprunter le terme de Barthes (1981 [1964], p. 258) sur le texte théâtrale, donne à ce roman l'équivalent romanesque de la théâtralité : l'ouvrage serait incomplet, imparfait, sans l'introduction du lecteur (et d'autres personnages extradiégétiques) dans son univers de narration polyvalent. 


\section{BIBLIOGRAPHIE}

Barthes, Roland. Essais critiques. Éditions du Seuil, Paris, 1981 [1963].

Collectif. Communications, 8: L'analyse structurale du récit. R. Barthes et al. L'École Pratique des Hautes Études, Paris, 1966. Éditions du Seuil, Paris, 1981.

Collectif. Théorie de la littérature: Textes des Formalistes russes. Éditions du Seuil, Paris, 1965.

Courtés, J., et A. J. Greimas. Dictionnaire raisonné de la théorie du langage. Hachette, Paris, 1979.

Eco, Umberto. La Struttura assente. Tascabili Bompiani, Milan, 1968.

Genette, G. Figures III. Collection Seuil Poétique, Paris, 1972.

—. «Frontières du récit» dans Communications, 8: L'analyse structurale du récit. Seuil, Paris, 1981.

Greimas, A. J. Du sens : Essais sémiotiques. Éditions du Seuil, Paris, 1970.

- Du sens II : Essais sémiotiques. Éditions du Seuil, Paris, 1976.

Maalouf, A. Le Rocher de Tanios. Éditions Grasset, Paris, 1993.

Marivaux, N. C. Le Paysan parvenu. Éditions Garnier Flammarion, Paris, 1965.

Poulet, Georges. La distance intérieure. Librairie Plon, Paris, 1952.

Rousset, Jean. Forme et signification. Librairie José Corti, Paris, 1962.

Todorov, Tzvetan. Introduction à la littérature fantastique. Éditions du Seuil, Paris, 1970. 